\title{
Quantification of optical absorption coefficients from acoustic spectra with photoacoustic tomography
}

Zijian Guo, Song Hu, Christopher P. Favazza, Todd N. Erpelding, Ladislav Jankovic, et al.

Zijian Guo, Song Hu, Christopher P. Favazza, Todd N. Erpelding, Ladislav Jankovic, Lihong V. Wang, "Quantification of optical absorption coefficients from acoustic spectra with photoacoustic tomography," Proc. SPIE 7899, Photons Plus Ultrasound: Imaging and Sensing 2011, 78990W (17 February 2011); doi: $10.1117 / 12.875015$

SPIE. Event: SPIE BiOS, 2011, San Francisco, California, United States 


\title{
Quantification of optical absorption coefficients from acoustic spectra with photoacoustic tomography
}

\author{
Zijian Guo ${ }^{\mathrm{a}}$, Song Hu $\mathrm{u}^{\mathrm{a}}$, Christopher P. Favazza ${ }^{\mathrm{a}}$, Todd N. Erpelding ${ }^{\mathrm{b}}$, Ladislav Jankovic ${ }^{\mathrm{b}}$, and \\ Lihong V. Wang*a \\ ${ }^{a}$ Optical Imaging Laboratory, Dept. of Biomedical Engineering, Washington University in St. Louis, \\ 1 Brookings Dr., St. Louis, MO, USA 63130 \\ ${ }^{b}$ Philips Research North America, 345 Scarborough Rd., Briarcliff Manor, NY, USA 10510; \\ *Corresponding author. Email: $\underline{\text { hwang@ } @ \text { biomed.wustl.edu }}$
}

\begin{abstract}
Optical absorption is closely associated with many physiologically important parameters, such as the concentration and oxygen saturation of hemoglobin, and it can be used to quantify the concentrations of non-fluorescent molecules. We introduce a method to quantify the absolute optical absorption based upon the acoustic spectra of photoacoustic (PA) signals. This method is self-calibrating and thus insensitive to variations in optical fluence. Factors such as the detection system bandwidth and acoustic attenuation can affect the quantification but can be canceled by measuring the acoustic spectra at two optical wavelengths. This method has been implemented on various PA systems, including optical-resolution PA microscopy, acoustic-resolution PA microscopy, and reconstruction based PA array systems. We quantified the optical absorption coefficients of phantom samples at various wavelengths. We also quantified the oxygen saturation of hemoglobin in live mice.
\end{abstract}

Keywords: Photoacoustic tomography, acoustic spectrum, optical absorption coefficient, blood oxygen saturation

\section{INTRODUCTION}

Total, oxygenated, and deoxygenated hemoglobin concentrations ([HbT], $\left[\mathrm{HbO}_{2}\right]$, and $\left.[\mathrm{HbR}]\right)$ are fundamental pathophysiological parameters in biomedicine. For example, abnormally low [HbT] may be caused by loss of blood, nutritional deficiency, chemotherapy, inflammation, kidney failure or bone marrow problems, while abnormally high $[\mathrm{HbT}]$ may be related to exposure to high altitude, smoking, dehydration and tumors. Blood oxygen saturation $\left(\mathrm{sO}_{2}\right)$, which is defined as $\left[\mathrm{HbO}_{2}\right]$ divided by $[\mathrm{HbT}]$, is vital in understanding brain hemodynamics in response to sensory stimulations ${ }^{1}$, monitoring healing of burns ${ }^{2}$ and wounds ${ }^{3}$, and evaluating the effectiveness of chemotherapy and radiotherapy on tumors ${ }^{4}$. Several techniques have been developed to quantify hemoglobin concentration and $\mathrm{sO}_{2}$ in vivo, including near-infrared spectroscopy (NIRS), blood oxygen level dependent (BOLD) contrast magnetic resonance imaging (MRI), electron paramagnetic resonance imaging (EPRI), positron emission tomography (PET), and single photon emission computed tomography (SPECT). However, all of these modalities have disadvantages: for example, poor spatial resolution, relative quantification, or undesirable contrast agent injection ${ }^{5,6}$.

\subsection{Photoacoustic tomography}

Photoacoustic (PA) tomography (PAT) has already demonstrated its ability to monitor biological hemodynamic functions without using exogenous contrast agents ${ }^{7-10}$. In PA imaging, the sample is illuminated, usually by a pulsed laser, and following the absorption of optical energy, an initial pressure is generated via thermo-elastic expansion. The PA waves then propagate and are detected by ultrasonic sensors. The strength of the initial pressure is directly proportional to the absorbed optical energy in the tissue, and therefore multi-wavelength PA measurements can yield optical absorption spectral information ${ }^{11}{ }^{12}$. Since ultrasound scattering is much weaker than optical scattering in biological tissues, PAT is capable of high resolution imaging at depths beyond the optical transport mean free path ${ }^{13}$. Moreover, because PA imaging inherently exploits optical absorption contrast, it usually has a higher sensitivity to optical absorption than other optical imaging technologies ${ }^{14}$. By measuring the optical absorption spectrum with PA imaging, we can potentially quantify $\mathrm{sO}_{2}$ in the same way NIRS does ${ }^{15}$, with high spatial resolution and sensitivity.

Photons Plus Ultrasound: Imaging and Sensing 2011, edited by Alexander A. Oraevsky, Lihong V. Wang, Proc. of SPIE Vol. 7899, 78990W · (C) 2011 SPIE · CCC code: 1605-7422/11/\$18 - doi: 10.1117/12.875015 


\subsection{Challenges in quantitative PAT}

PA images are spatial mappings of the absorbed optical energy $A(\vec{r})\left(\mathrm{Jm}^{-3}\right)$, which is the product of the optical absorption coefficient $\mu_{a}(\vec{r})\left(\mathrm{m}^{-1}\right)$ and the fluence $F(\vec{r})\left(\mathrm{Jm}^{-2}\right)$ at location $\vec{r}$. To obtain the intrinsic tissue property $\mu_{a}(\vec{r})$, we must somehow compensate for the extrinsic quantity $F(\vec{r})$. Since the optical parameters for tissues are usually heterogeneous and unknown, the fluence varies from case to case and is difficult to model. As a consequence, compensating for the fluence in quantitative in vivo PAT is a challenge ${ }^{6}{ }^{16}$. Currently, fluence compensation can be done invasively ${ }^{5,7,17,18}$ or non-invasively ${ }^{19,20}$. The invasive method puts an optical absorber with known spectrum close to the region of interest, and then normalizes the measured PA signals from the objects with the amplitudes of the PA signals from the absorber. Alternatively, the incident fluence can be estimated by measuring the light transmission through a sample of excised tissue of the same type as the tissue overlaying the region of interest. The non-invasive method involves solving the radiative transfer equation (RTE) and PA wave equations with iterative algorithms. All of these methods are based on the linear relationship between the local fluence incident on the blood vessel, acquired either by experimental measurements or numerical simulation, and the peak amplitude of the PA signal. The temporal profile of the PA signal was also used to quantify the optical absorption coefficients ${ }^{21-24}$. This temporal method is self-calibrating since it depends on the relative temporal profile rather than the absolute amplitude of the PA signal. Therefore, it is less dependent on changes in the optical properties of overlying tissues. However, since the temporal profile is distorted by various factors, such as the limited bandwidth of ultrasonic detectors and the frequencydependent acoustic attenuation, directly fitting the temporal profile for the optical absorption may be inaccurate.

We propose to use the acoustic spectra of the PA signals to quantify optical absorption. By carefully investigating the factors involved in generating the acoustic spectrum, the effects of detector bandwidth and acoustic attenuation are eliminated, as shown later. This method is also self-calibrating since it deals only with the relative change in various acoustic frequencies. Therefore, it is insensitive to fluence fluctuations.

\section{METHODS}

\subsection{Quantification of optical absorption coefficients from acoustic spectra}

The acoustic spectrum $S(\omega)$ of the received PA signal is a function of acoustic frequency $\omega$. It depends on three factors: 1) the 'real' object spectrum $O(\omega)$ measured with unit fluence, which is related to the target object's shape, size, optical properties, and fluence incident directions; 2) the system dependent response $H(\omega)$, which is the Fourier transform of the PA signal from an ideal point absorber with an absorption coefficient of $\mu_{a}(\lambda)$ measured with this system; 3) the tissue related acoustic attenuation effect $a(\omega)$, which is related to the acoustic properties of the tissue that lies between the target objects and the detector. Based on the system linearity assumption, we have $S(\omega)=F(\lambda) O(\omega) H(\omega) a(\omega)$, where $F(\lambda)$ represents the optical-wavelength- $\lambda$-dependent incident fluence on the absorbing target. The last two terms remain unchanged when samples are measured with the same system under the same condition, and therefore are usually cancellable. Here the same condition means the acoustic properties of the tissue between the sample and the detectors are the same. An obvious example is where various optical wavelengths are used to excite one blood vessel. Therefore, simply dividing the PA acoustic spectrum measured at one optical wavelength by the spectrum measured at another wavelength, we can eliminate the system dependent effects and the acoustic attenuation effect.

\subsection{Implementation of the method in the optical ballistic regime}

As the first feasibility study ${ }^{25}$, we validated this idea using one form of PA imaging, optical resolution photoacoustic microscopy (OR-PAM) ${ }^{26}$, where the object spectrum $O(\omega)$ can be relatively easily modeled. In ORPAM, PA A-scan signals are acquired through time-resolved ultrasonic detection, and three-dimensional (3D) images were formed by raster scanning the ultrasonic transducer along the transverse plane [Fig. 1(a)]. The axial resolution of the system depended on the ultrasonic transducer bandwidth (centered at $50 \mathrm{MHz}$ with $80 \%$ bandwidth), while the lateral resolution relied on optical focusing, which can reach the theoretical optical diffraction limit. For the system we used, the axial and lateral resolutions were quantified to be $15 \mu \mathrm{m}$ and $5 \mu \mathrm{m}$, respectively. Therefore, the surface of blood 
vessels with $>30 \mu \mathrm{m}$ diameter can be roughly treated as a flat surface. In this case, the acoustic spectrum of the generated PA signal is only related to the optical penetration depth. If we use $F_{0}$ to denote the incident fluence on the surface of the blood vessel, the fluence inside the blood vessel obeys Beer's law and can be written as $F(z)=F_{0} \exp \left(-\mu_{a} z\right)$ [Fig. 1(b)]. Here the reduced scattering coefficient is neglected, since $\mu_{s}{ }^{\prime}$ is much smaller than $\mu_{a}$ in blood in the optical spectral region typically used ${ }^{27}$. The PA signal generated by the object can be written as

$$
O(t)=\mu_{a} \exp \left(-\mu_{a} c t\right),(1)
$$

where $c$ is the speed of sound in the biological tissue. Fourier transformation of Eq. (1) leads to

$$
|O(\omega)|=\frac{1}{\sqrt{\left(\omega / \mu_{a}\right)^{2}+c^{2}}} .
$$

If the PA signals of the blood vessel are measured at two optical wavelengths, the ratio of the spectra of the PA signals can be written as

$$
\frac{S_{1}(\omega)}{S_{2}(\omega)}=\frac{F_{0}\left(\lambda_{1}\right) O_{1}(\omega) H(\omega) a(\omega)}{F_{0}\left(\lambda_{2}\right) O_{2}(\omega) H(\omega) a(\omega)}=\frac{F_{0}\left(\lambda_{1}\right) \sqrt{\left(\omega / \mu_{a 2}\right)^{2}+c^{2}}}{F_{0}\left(\lambda_{2}\right) \sqrt{\left(\omega / \mu_{a 1}\right)^{2}+c^{2}}} .
$$

By fitting this ratio we can derive the absolute values of $\mu_{a 1}, \mu_{a 2}$, and $F_{0}\left(\lambda_{1}\right) / F_{0}\left(\lambda_{2}\right)$.

(a)

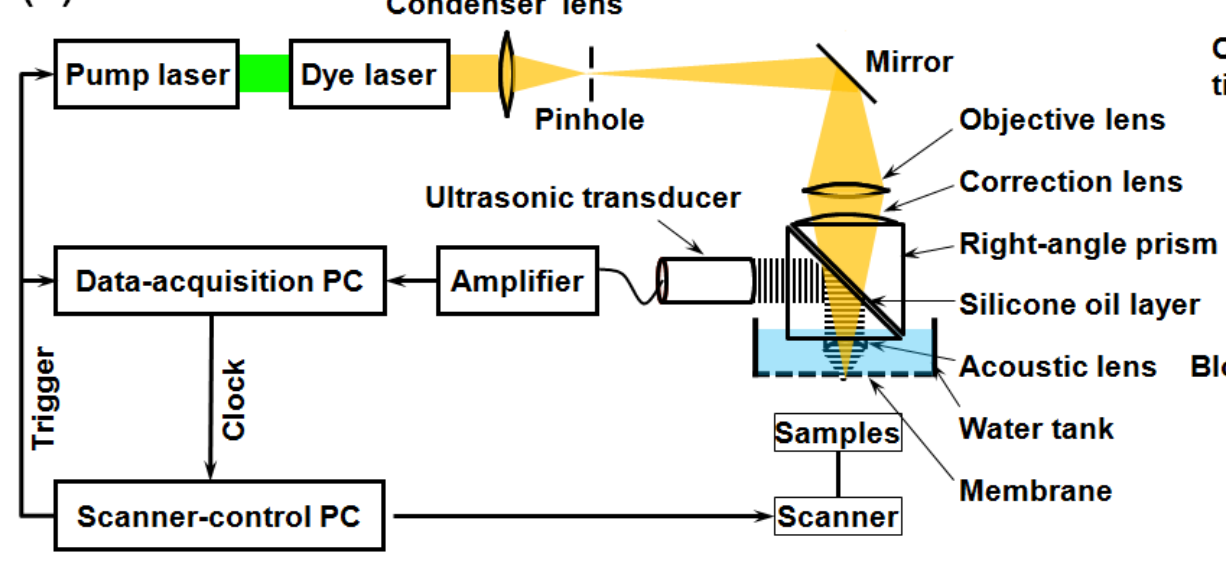

(b)

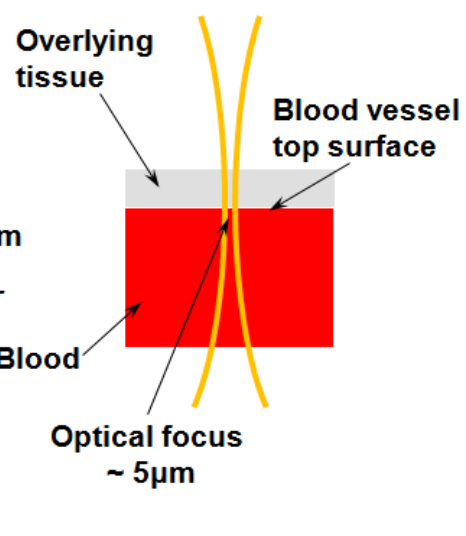

Figure 1. Schematic of the OR-PAM system and experimental setup. (a) The schematic of the OR-PAM system. (b) The optical focus is much smaller than the targeted blood vessel, whose top surface within the optical focal zone can therefore be approximated as a plane.

\subsection{Implementation of the method in the optical diffusive regime}

We further applied this idea to acoustic resolution PA microscopy (AR-PAM) ${ }^{9}$. Figure 2(a) shows the PAM setup. A dye laser pumped by a Nd:YLF laser was the irradiation source. AR-PAM acquires 3D images by raster scanning a focused ultrasonic transducer. The lateral resolution of AR-PAM is determined by the focal diameter of the ultrasonic transducer $(\sim 45 \mu \mathrm{m}$ for the $50 \mathrm{MHz}$ transducer). With AR-PAM, several assumptions can simplify the modeling of $O(\omega, \lambda)$. First, the surface of blood vessels with diameters much larger than the lateral resolution can be roughly treated as a flat surface. Second, when imaging blood vessels at depths greater than one transport mean free path $\left(l_{t}{ }^{\prime} \sim 1 \mathrm{~mm}\right.$ in biological tissue), we can further assume that the light is completely diffused and thus the incident angles range randomly from 0 to $\pi$ [Fig. 2(b)]. As such, $O(\omega, \lambda)$ is related only to the optical properties of the target blood vessel. If $\mu_{a}\left(\lambda_{1}\right)>\mu_{a}\left(\lambda_{2}\right)$, light penetrates shallower at $\lambda_{1}$ and deeper at $\lambda_{2}$ in the blood vessel. In other words, the 
fluence decays faster at $\lambda_{1}$, and therefore the produced PA signal is also sharper in the time domain. As a consequence, $O\left(\omega, \lambda_{1}\right)$, the Fourier transform of the PA time domain signal acquired at $\lambda_{1}$, contains more high frequency components than $O\left(\omega, \lambda_{2}\right)$. Mathematically speaking, if $\mu_{a}\left(\lambda_{1}\right)>\mu_{a}\left(\lambda_{2}\right)$,

$$
O\left(\omega+\omega_{0}, \lambda_{1}\right) / O\left(\omega, \lambda_{1}\right)>O\left(\omega+\omega_{0}, \lambda_{2}\right) / O\left(\omega, \lambda_{2}\right),
$$

where $\omega_{0}$ is an arbitrary positive frequency that divides the spectrum into a high frequency band $\omega_{0}<\omega<2 \omega_{0}$ and a low frequency band $0<\omega<\omega_{0}$. Eq. (4) is equivalent to

$$
S\left(\omega+\omega_{0}, \lambda_{1}\right) / S\left(\omega, \lambda_{1}\right)>S\left(\omega+\omega_{0}, \lambda_{2}\right) / S\left(\omega, \lambda_{2}\right)
$$

since $F(\lambda), a(\omega)$, and $H(\omega)$ can be canceled. We define $R(\lambda)=\int_{0}^{\omega_{0}} S\left(\omega+\omega_{0}, \lambda\right) / S(\omega, \lambda) d \omega$. If $\mu_{a}\left(\lambda_{1}\right)>\mu_{a}\left(\lambda_{2}\right)$, then $R\left(\lambda_{1}\right)>R\left(\lambda_{2}\right)$, and vice versa. Therefore, we can rank $\mu_{a}(\lambda)$ based on $R(\lambda)$ despite the optical fluence decay and the aforementioned acoustic effects. If we select appropriate wavelengths, $\mathrm{sO}_{2}$ can be quantified from the ranking of $\mu_{a}(\lambda)$. If $\mu_{a}\left(\lambda_{1}\right)>\mu_{a}\left(\lambda_{2}\right)$, then

$$
\left[\varepsilon_{\mathrm{HbR}}\left(\lambda_{1}\right)-\varepsilon_{\mathrm{HbR}}\left(\lambda_{2}\right)\right]+\left[\varepsilon_{\mathrm{HbO}_{2}}\left(\lambda_{1}\right)-\varepsilon_{\mathrm{HbO}_{2}}\left(\lambda_{2}\right)-\varepsilon_{\mathrm{HbR}}\left(\lambda_{1}\right)+\varepsilon_{\mathrm{HbR}}\left(\lambda_{2}\right)\right] \mathrm{sO}_{2}>0,
$$

where $\varepsilon_{\mathrm{HbR}}(\lambda)$ and $\varepsilon_{\mathrm{HbO}_{2}}(\lambda)$ are the known molar extinction coefficients $\left(\mathrm{m}^{-1} \mathrm{M}^{-1}\right)$ of deoxyhemoglobin $\mathrm{HbR}$ and oxyhemoglobin $\mathrm{HbO}_{2}$ at wavelength $\lambda$. As a result, the range of $\mathrm{sO}_{2}$ can be quantified from Eq. (6).

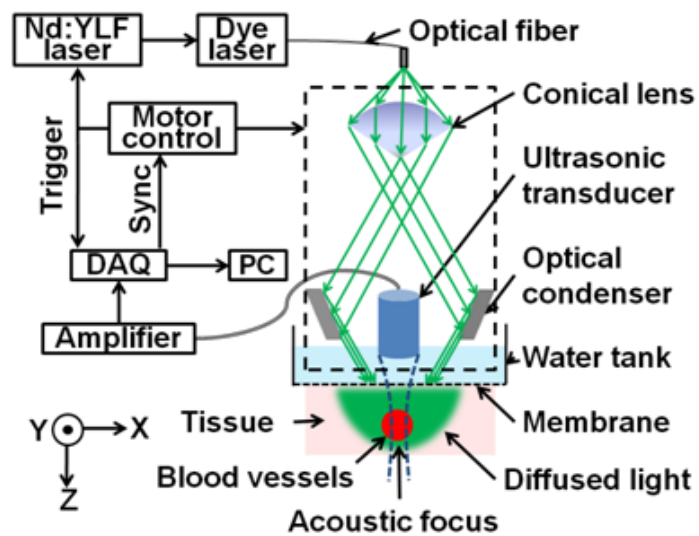

(a)

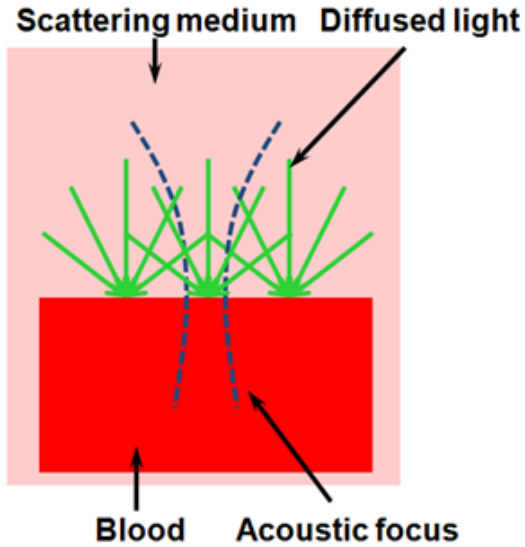

(b)

Figure 2. Schematic of the AR-PAM system and phantom experiment setups. (a) The schematic of the AR-PAM system. A dye laser pumped by a Nd:YLF laser was the irradiation source. The laser beam from the dye laser was delivered through an optical fiber and passed through a conical lens to provide a ring-shaped area of illumination. A focused ultrasonic transducer was employed to detect PA waves. (b) AR-PAM system was used to image a planar blood phantom buried $1.5 \mathrm{~mm}$ deep in the optical scattering medium.

This method can also be applied on the reflection-mode reconstruction-based PAT array systems ${ }^{28}$. Figure 3(a) shows the system setup. Light from a tunable dye laser pumped by a Nd:YAG laser was delivered through a fiber bundle. Raw PA and ultrasonic (US) RF data are acquired with a clinical US imaging system (iU22; Philips Healthcare, Andover, MA), and transferred to a custom-built data acquisition system. By employing the synthetic aperture focusing technique, we can use virtual acoustic focuses to mimic the physical acoustic focus in AR-PAM [Fig. 3(b)]. As such, we can make similar assumptions about the object geometry and the illumination condition, and $O(\omega, \lambda)$ is related only to the optical properties of the target blood vessel. Therefore, we can quantify $\mathrm{sO}_{2}$ in the same way as in AR-PAM. 


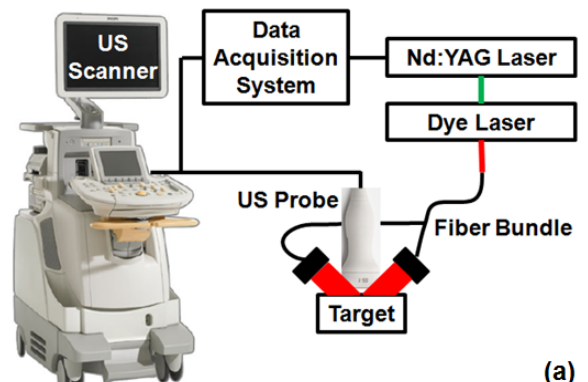

(a)

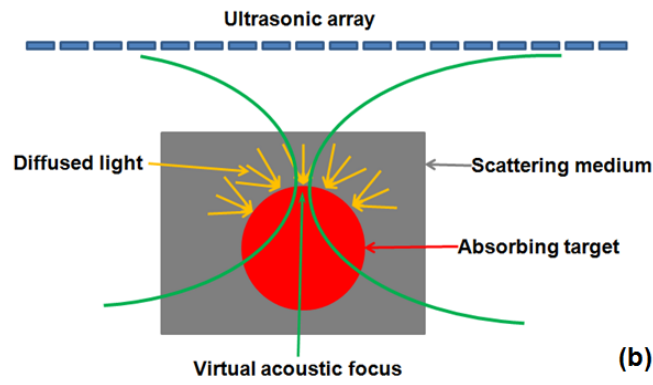

(b)

Figure 3. Schematic of the ultrasonic array system and experiment setup. (a) The schematic of the ultrasonic array system. A dye laser pumped by a Nd:YAG laser was used as the irradiation source. The laser beam from the dye laser was delivered through an optical fiber bundle. An ultrasonic array was employed to detect PA waves. (b) The ultrasonic array system was used to image a phantom buried in the optical scattering medium.

\section{RESULTS AND DISCUSSION}

\subsection{Quantification of the absorption coefficients with OR-PAM}

In a phantom study, the original black ink was diluted with water in six ratios ranging from 1:1 to 1:6. The original and diluted ink samples were sequentially placed in a container, sealed with plastic membrane, and then the container was placed in a water tank. PA A-line signals were acquired from these samples, and the acoustic spectra of the PA signals are shown in Fig. 4(a). Compared with the spectrum of the PA signal from the original ink sample, the spectra of the PA signals from the diluted ink samples are "shifted" to lower frequencies. Light penetrated deeper in lower concentration ink samples, and the corresponding PA signals decay more slowly in the time domain. Therefore, the spectra contain more low-frequency components. By dividing the measured spectra of any two ink samples frequency-by-frequency [Fig. 4(b)], we can find the absorption coefficients of both samples by fitting the resultant ratio curve with Eq. (3). Because parts of the spectra (grey bands) are unreliable due to the limited bandwidth detection, they are not used for the fitting. By pairing the spectrum from the 1:1 diluted ink sample with the spectra from other six samples, we quantified the absorption coefficients of all seven samples. The quantified values and their theoretical predictions are plotted in Fig. 4(c). The error bars indicate the fitting standard deviations.
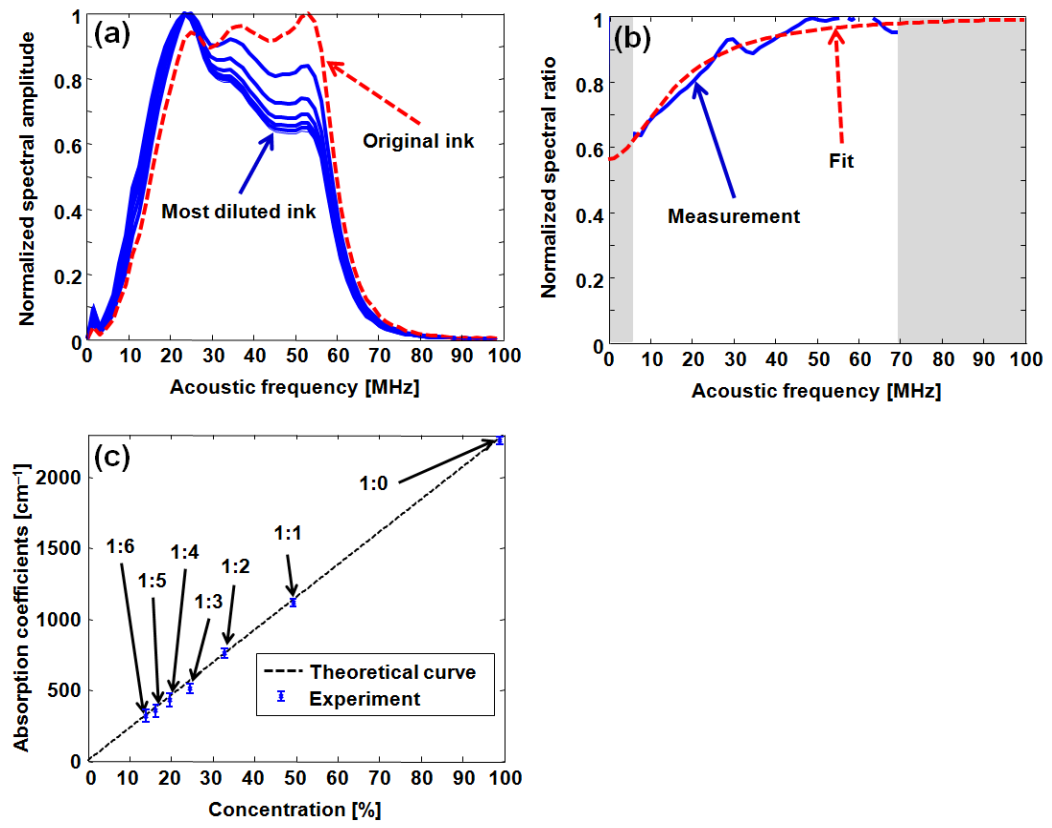

Figure 4. Quantifying the optical absorption coefficients of ink. (a) Acoustic spectra of PA signals from original and diluted black ink samples. (b) Ratio of the acoustic spectral amplitudes of PA signals from two samples and fitting with the theoretical formula. (c)

Fitting result with 7 phantoms. 


\subsection{Quantification of the absorption coefficients with AR-PAM}

We used AR-PAM to image an oxygenated bovine blood phantom with four wavelengths $(560 \mathrm{~nm}, 565 \mathrm{~nm}$, $570 \mathrm{~nm}$, and $575 \mathrm{~nm}$ ), where the absorption coefficients of the oxygenated blood monochromatically increase $\left[\mu_{a}(560)<\mu_{a}(565)<\mu_{a}(570)<\mu_{a}(575)\right]$. The blood phantom was placed in a container, and sealed with plastic membrane, and then the container was buried in an optical scattering medium (10\% gelatin, $1 \%$ intralipid, $1.5 \mathrm{~mm}$ thick on top) [Fig. 2(b)]. PA A-line signals at four wavelengths were acquired from the samples [Fig. 5(a)], and the corresponding acoustic spectra of the PA signals $[S(\omega, \lambda)]$ were calculated [Fig. 5(b)]. As shown in Fig. 5(b), the acoustic spectra 'shift' to higher frequencies as the wavelengths increase, because the absorption coefficients increase. We further quantified the shift by calculating $R(\lambda)$, as shown in Fig. 5(c). The ranking results agree well with the spectrophotometer measurements. Using the peaks of the amplitudes to quantify the absorption coefficients is inaccurate [Fig. 5(c)], because of the wavelength-dependent fluence decay introduced by the scattering medium.

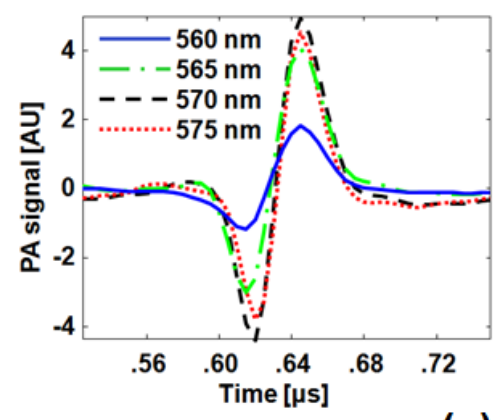

(a)

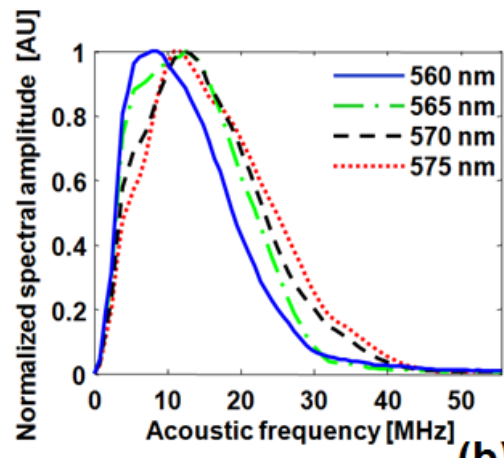

(b)

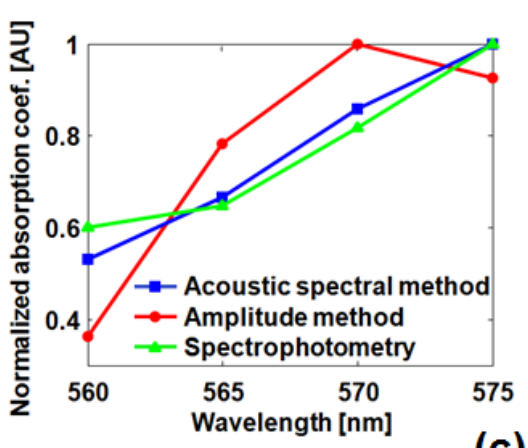

(c)

Figure 5. Quantifying the optical absorption coefficients of oxygenated bovine blood phantom. (a) PA time domain A-scan signals of the planar oxygenated bovine blood phantom buried in the scattering medium. (b) Acoustic spectra of the PA A-scan signals. (c) Comparison of the quantification results from the amplitude method, the acoustic spectra method, and the spectrophotometry.

\subsection{Quantification of the absorption coefficients with the PAT array system}

We used the Philips array system to image a copper chloride phantom with eight wavelengths $(610 \mathrm{~nm}-680$ $\mathrm{nm}$ ), where the absorption coefficients of copper chloride monochromatically increase. In the phantom experiment, the copper chloride solution was placed in a container, and sealed with plastic membrane, and then the container was buried in an optical scattering medium (10\% gelatin, $1 \%$ intralipid, $2 \mathrm{~cm}$ thick on top). A graphite rod was buried under the scattering medium to correct for the fluence. The PA image at $610 \mathrm{~nm}$ is shown in Fig. 6(a). PA A-line signals at eight wavelengths were acquired from the samples, and the corresponding acoustic spectra of the PA signals $[S(\omega, \lambda)]$ were calculated [Fig. 6(b)]. As shown in Fig. 6(b), the acoustic spectra 'shift' to higher frequencies as the wavelengths increase, because the absorption coefficients increase. We further quantified the shift by calculating $R(\lambda)$, as shown in Fig. 6(c). The quantification results agree well with the spectrophotometer measurements. Using the peaks of the amplitudes to quantify the absorption coefficients is inaccurate without fluence compensation [Fig. 6(c)], because of the wavelength-dependent fluence decay introduced by the scattering medium. 


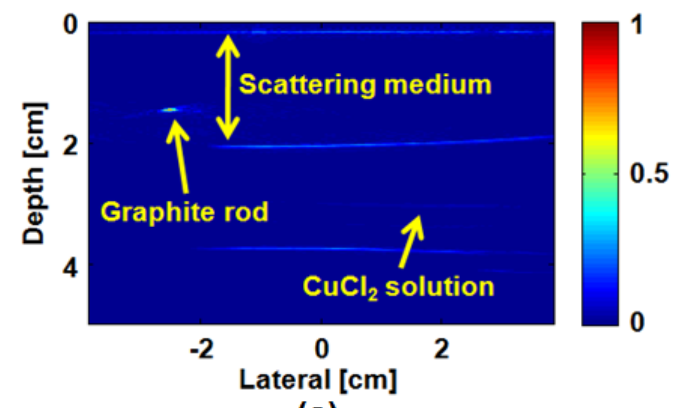

(a)
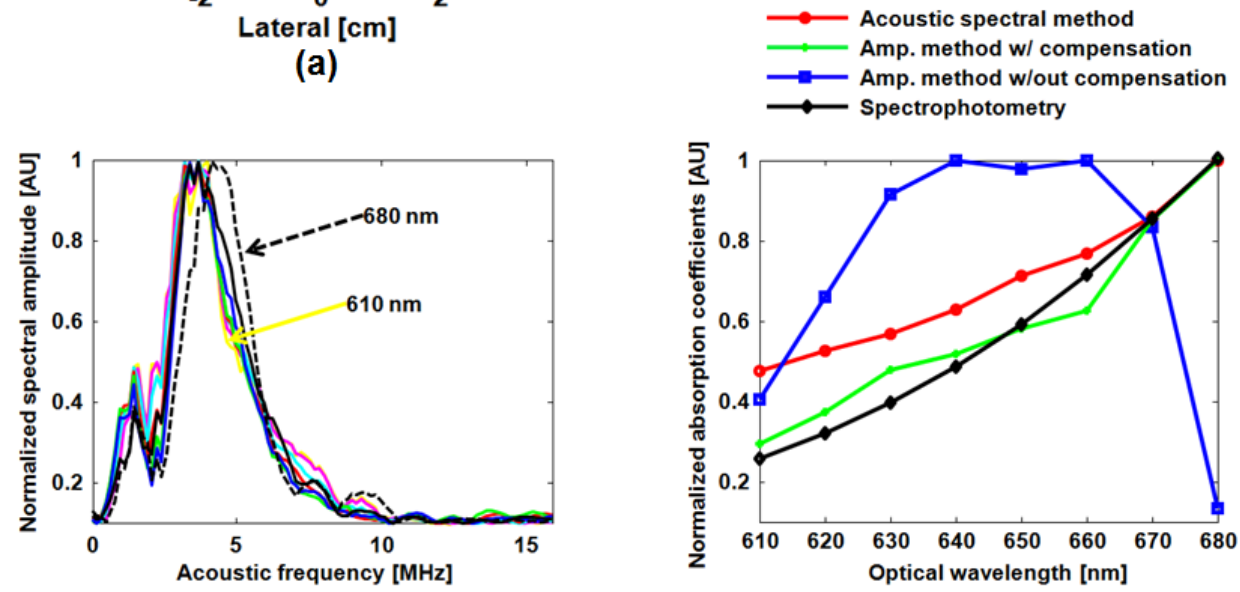

Figure 6. Quantifying the optical absorption coefficients of copper chloride phantom. (a) PA image of the copper chloride phantom buried in the scattering medium. (b) Acoustic spectra of the PA A-scan signals. (c) Comparison of the quantification results from the amplitude method, the acoustic spectra method, the amplitude method with fluence compensation, and spectrophotometry.

\subsection{In vivo experiment}

In an in vivo experiment on AR-PAM, we imaged a 5-mm by 5-mm region of the back of a nude mouse with five optical wavelengths. Figure 7(a) shows the PA maximum amplitude projection (MAP) image acquired with an optical wavelength of $575 \mathrm{~nm}$. Each point in the MAP image corresponds to the maximum value of a Hilbert transformed PA A-line. At each of the scanning locations, we have A-lines at five wavelengths. Based on the multiwavelength A-lines, we calculated $R(\lambda)$, from which we can obtain the ranking of $\mu_{a}(\lambda)$. In non-blood vessel regions, the ranking appears to be completely random. Therefore, we first threshold out the non-blood vessel regions and then quantify the $\mathrm{sO}_{2}$ ranges using Eq. (8), with the results shown in Fig. 7(b). We then quantify $\mathrm{sO}_{2}$ in the same way, but using the amplitude ranking method [Fig. 7(c)]. The quantification is inaccurate, as the in vivo $\mathrm{SO}_{2}$ in arteries should be between $90-100 \%$ under normal conditions, because the amplitude ranking does not represent the ranking of $\mu_{a}(\lambda)$.
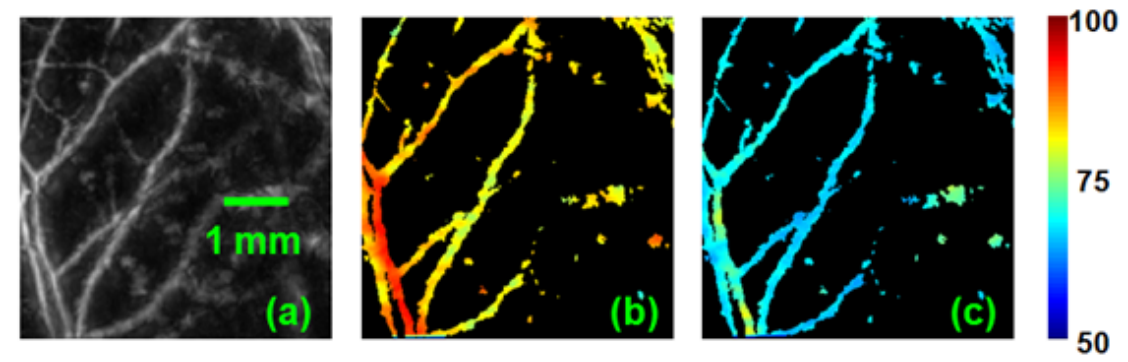

Figure 7. Quantifying the $\mathrm{sO}_{2}$ of blood vessels in the back of a nude mouse in vivo. (a) MAP image acquired at $575 \mathrm{~nm}$. (b) $\mathrm{sO}_{2}$ image quantified with the acoustic spectra method. (c) $\mathrm{sO}_{2}$ image quantified with the amplitude method. 


\section{CONCLUSIONS}

We introduce a method to quantify the absolute optical absorption based upon the acoustic spectra of photoacoustic signals. This method is self-calibrating and thus insensitive to variations in optical fluence. Factors such as the detection system bandwidth and acoustic attenuation can affect the quantification but can be canceled by measuring the acoustic spectra at two optical wavelengths. We quantified the optical absorption coefficients phantom samples at various wavelengths. We also quantified the oxygen saturation of hemoglobin in live mice.

\section{REFERENCES}

[1] I. Vanzetta and A. Grinvald, "Increased cortical oxidative metabolism due to sensory stimulation: Implications for functional brain imaging," Science 286(5444), 1555-1558 (1999)

[2] B. Venkatesh, R. Meacher, M. J. Muller, T. J. Morgan and J. Fraser, "Monitoring tissue oxygenation during resuscitation of major burns," Journal of Trauma-Injury Infection and Critical Care 50(3), 485-493 (2001)

[3] A. A. Tandara and T. A. Mustoe, "Oxygen in wound healing - More than a nutrient," World Journal of Surgery 28(3), 294-300 (2004)

[4] C. Menon and D. L. Fraker, "Tumor oxygenation status as a prognostic marker," Cancer Letters 221(2), 225-235 (2005)

[5] H. F. Zhang, K. Maslov, M. Sivaramakrishnan, G. Stoica and L. H. V. Wang, "Imaging of hemoglobin oxygen saturation variations in single vessels in vivo using photoacoustic microscopy," Applied Physics Letters 90(5), 053901 (2007)

[6] M. Sivaramakrishnan, K. Maslov, H. F. Zhang, G. Stoica and L. V. Wang, "Limitations of quantitative photoacoustic measurements of blood oxygenation in small vessels," Physics in Medicine and Biology 52(5), 1349-1361 (2007)

[7] X. D. Wang, X. Y. Xie, G. N. Ku and L. H. V. Wang, "Noninvasive imaging of hemoglobin concentration and oxygenation in the rat brain using high-resolution photoacoustic tomography," Journal of Biomedical Optics 11(2), 024015 (2006)

[8] X. D. Wang, Y. J. Pang, G. Ku, X. Y. Xie, G. Stoica and L. H. V. Wang, "Noninvasive laser-induced photoacoustic tomography for structural and functional in vivo imaging of the brain," Nature Biotechnology 21(7), 803-806 (2003)

[9] H. F. Zhang, K. Maslov, G. Stoica and L. H. V. Wang, "Functional photoacoustic microscopy for high-resolution and noninvasive in vivo imaging," Nature Biotechnology 24(7), 848-851 (2006)

[10] K. M. Stantz, B. Liu, M. Cao, D. R. Reinecke, K. Miller and R. A. Kruger, "Photoacoustic spectroscopic imaging of intra-tumor heterogeneity and molecular identification," Proc. SPIE 6086, 608605 (2006).

[11] M. H. Xu and L. H. V. Wang, "Photoacoustic imaging in biomedicine," Review of Scientific Instruments 77(4), 041101 (2006).

[12] C. Li and L. H. V. Wang, "Photoacoustic tomography and sensing in biomedicine," Physics in Medicine and Biology 54(19), R59-R97 (2009)

[13] L. V. Wang and H. Wu, [Biomedical Optics: Principles and Imaging], Wiley, Hoboken, NJ, 466-500 (2007).

[14] L. V. Wang, "Tutorial on photoacoustic microscopy and computed tomography," Ieee Journal of Selected Topics in Quantum Electronics 14(1), 171-179 (2008)

[15] B. Chance, E. Borer, A. Evans, G. Holtom, J. Kent, M. Maris, K. Mccully, J. Northrop and M. Shinkwin, "Optical and Nuclear-Magnetic-Resonance Studies of Hypoxia in Human Tissue and Tumors," Annals of the New York Academy of Sciences 551, 1-16 (1988)

[16] B. T. Cox, J. G. Laufer and P. C. Beard, "The challenges for quantitative photoacoustic imaging," Proc. SPIE 7177 , 717713 (2009)

[17] K. Maslov, H. F. Zhang and L. V. Wang, "Effects of wavelength-dependent fluence attenuation on the noninvasive photoacoustic imaging of hemoglobin oxygen saturation in subcutaneous vasculature in vivo," Inverse Problems 23(6), S113-S122 (2007)

[18] H. F. Zhang, K. Maslov and L. H. V. Wang, "In vivo imaging of subcutaneous structures using functional photoacoustic microscopy," Nature Protocols 2(4), 797-804 (2007) 
[19] J. Laufer, D. Delpy, C. Elwell and P. Beard, "Quantitative spatially resolved measurement of tissue chromophore concentrations using photoacoustic spectroscopy: application to the measurement of blood oxygenation and haemoglobin concentration," Physics in Medicine and Biology 52(1), 141-168 (2007)

[20] Z. Yuan and H. B. Jiang, "Quantitative photoacoustic tomography: Recovery of optical absorption coefficient maps of heterogeneous media," Applied Physics Letters 88(23), 231101 (2006)

[21] R. O. Esenaliev, I. V. Larina, K. V. Larin, D. J. Deyo, M. Motamedi and D. S. Prough, "Optoacoustic technique for noninvasive monitoring of blood oxygenation: a feasibility study," Applied Optics 41(22), 4722-4731 (2002)

[22] J. Laufer, C. Elwell, D. Delpy and P. Beard, "In vitro measurements of absolute blood oxygen saturation using pulsed near-infrared photoacoustic spectroscopy: accuracy and resolution," Physics in Medicine and Biology 50(18), 4409-4428 (2005)

[23] Y. Y. Petrov, I. Y. Petrova, I. A. Patrikeev, R. O. Esenaliev and D. S. Prough, "Multiwavelength optoacoustic system for noninvasive monitoring of cerebral venous oxygenation: a pilot clinical test in the internal jugular vein," Optics Letters 31(12), 1827-1829 (2006)

[24] Y. Wang and R. K. Wang, "Photoacoustic recovery of an absolute optical absorption coefficient with an exact solution of a wave equation," Physics in Medicine and Biology 53(21), 6167-6177 (2008)

[25] Z. Guo, S. Hu and L. V. Wang, "Calibration-free absolute quantification of optical absorption coefficients using acoustic spectra in 3D photoacoustic microscopy of biological tissue," Optics Letters 35(12), 2067-2069 (2010)

[26] K. Maslov, H. F. Zhang, S. Hu and L. V. Wang, "Optical-resolution photoacoustic microscopy for in vivo imaging of single capillaries," Optics Letters 33(9), 929-931 (2008)

[27] A. Roggan, M. Friebel, K. Dorschel, A. Hahn and G. Muller, "Optical properties of circulating human blood in the wavelength range 400-2500 NM," Journal of Biomedical Optics 4(1), 36-46 (1999)

[28] T. N. Erpelding, C. Kim, M. Pramanik, L. Jankovic, K. Maslov, Z. Guo, J. A. Margenthaler, M. D. Pashley and L. H. V. Wang, "Sentinel Lymph Nodes in the Rat: Noninvasive Photoacoustic and US Imaging with a Clinical US System," Radiology 256(1), 102-110 (2010) 